\title{
BP-SY-3-1
}

\section{Overview of smart healthcare}

\author{
Young-Hak KIM ${ }^{*}$
}

Department of Cardiology, Information Medicine, University of Ulsan College of Medicine, Asan Medical Center, Seoul, Korea

Lecture: The 4th Industrial Revolution is described as "a variety of new technologies that integrate the physical, biological, and digital worlds based on big data and affect all fields such as economy and industry." In the new era, new types of products and businesses are being created based on innovative technologies such as artificial intelligence, big data, IoT, and mobile, and this has an overall impact on all fields, including economy and industry. In the era of the 4th industrial revolution, the healthcare industry was also combined and fused with the ICT industry (Information and Communication Technology), which created a new field called smart healthcare. Smart healthcare refers to the combination of ICT technology with the existing traditional hospital and doctor-centered medical industry, and provides more convenient and diverse types of medical services to consumers. Smart healthcare will revolutionize the medical industry in almost every field, including medical devices, new drug development, disease diagnosis and prediction, and improvement of hospital processes. There are still many obstacles such as security and effectiveness issues, but it is expected that it will be overcome by the efforts of the officials. In this lecture, I think it will be an opportunity to check the future and potential of the evolving healthcare field by grafting cutting-edge technologies. 